\title{
VELOCIDADE AUTOSSELECIONADA E IDEAL DA CAMINHADA DE AMPUTADOS TRANSFEMORAIS: SOLO E ESTEIRA
}

\author{
SELF-SELECTED AND OPTIMAL WALKING SPEED OF TRANSFEMORAL AMPUTEES: GROUNDVS. TREADMILL
}

VELOCIDAD AUTOSELECCIONADA E IDEAL EN LA MARCHA DE AMPUTADOS TRANSFEMORALES:

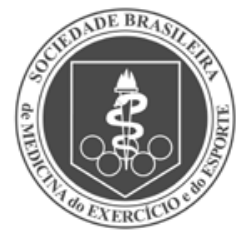

Artigo Original

ORIGINAL ARTICLE

Artículo Original

SUELO Y CINTA CAMINADORA

Renata Luísa Bona ${ }^{1,3}$

(Fisioterapeuta)

Natalia Andrea Gomeñuka

(Educadora Física)

Artur Bonezi dos Santos ${ }^{1,2}$

(Educador Físico)

Jorge Storniolo 4

(Educador Físico)

Paula Figueiredo da Silva ${ }^{3}$

(Educadora Física)

Leonardo Alexandre Peyré

Tartaruga² (Educador Físico)

1. Laboratorio de Investigación en Biomecánica y Análisis del Movimiento, Universidad de la Republica Uruguay, Centro Universitario de Paysandú, Paysandú, Uruguay.

2. Laboratório de Pesquisa do Exercício, Universidade Federal do Rio Grande do Sul, Porto Alegre, Brasil.

3. Laboratório de Pesquisa em Fisiopatologia de Exercício, Hospital de Clínicas de Porto Alegre, Porto Alegre, Brasil.

4. Physiomechanics of Locomotion Laboratory, Department of Human Physiology, Università degli Studi di Milano ou University of Milan. Milan, Italian.

\section{Correspondência:}

Laboratorio de Investigación en Biomecánica y Análisis del Movimiento, Universidad de la Republica Uruguay, Rua Florida 1065, Cidade de Paysandú, Uruguay. 60.000 .

renatalbona@gmail.com

\section{RESUMO}

Introdução: A velocidade de progressão é, em geral, determinada em pesquisas na área da locomoção. Objetivo: Comparar as medidas de velocidade autosselecionada no solo, na esteira rolante e a velocidade ideal estimada pelo número de Froude em sujeitos amputados transfemorais. Método: Primeiramente foi determinada a velocidade no solo; em seguida, realizou-se o teste na esteira, e a velocidade ideal foi estimada a partir dos dados antropométricos. Todos os sujeitos utilizavam joelho hidráulico e pé em fibra de carbono. Para comparação entre as velocidades foi realizada ANOVA de duas vias. Resultados: A velocidade autosselecionada na esteira foi menor (22\%) do que no solo. Tanto a velocidade autosselecionada na esteira como a do solo foram $44 \% \mathrm{e}$ $22 \%$ menores do que a velocidade ideal estimada, respectivamente. Conclusão: As velocidades analisadas no presente estudo foram diferentes, provavelmente, devido à variação dos parâmetros cinemáticos.

Descritores: medição de velocidade; amputados; caminhada.

\section{ABSTRACT}

Introduction: The speed of progression is generally determined in researches in the field of locomotion. Objective: To compare the self-selected velocity measurements on the ground, on treadmill and the optimal speed estimated by the Froude number in subjects with transfemoral amputation. Methods: First, the ground speed was determined; then the treadmill test was performed, and the optimal speed was estimated from anthropometric data. All subjects had hydraulic knee and carbon fiber foot. To compare the speeds, we used the two-way ANOVA. Results: The self-selected speed in the treadmill was lower (22\%) compared with the ground. Both the self-selected speed in treadmill as ground were $44 \%$ and 22\% lower than the estimated optimum speed, respectively. Conclusion: The speeds analyzed in this study were different, probably due to the variation of the kinematic parameters.

Keywords: velocity measurement; amputees; walking.

\section{RESUMEN}

Introducción: La velocidad de progresión es generalmente determinada en investigaciones en el ámbito de la locomoción. Objetivo: Comparar las mediciones de la velocidad autoseleccionada en el suelo, en la cinta caminadora y la velocidad ideal estimada por el número de Froude en sujetos con amputación transfemoral. Métodos: En primer lugar, se determinó la velocidad en el suelo; después, se realizó la prueba en la cinta caminadora, y la velocidad ideal fue estimada a partir de los datos antropométricos. Todos los sujetos tenían rodilla hidráulica y pie en fibra de carbono. Para comparar las velocidades, se utilizó el ANOVA de dos vías. Resultados: La velocidad autoseleccionada en la cinta caminadora fue menor (22\%) que en el suelo. Tanto la velocidad autoseleccionada en la cinta caminadora como la del suelo fueron $44 \%$ y $22 \%$ menores a la velocidad ideal estimada, respectivamente. Conclusión: Las velocidades analizadas en el presente estudio fueron diferentes, probablemente debido a la variación de los parámetros cinemáticos.

Descriptores: medición de velocidades; amputados; caminata.

\section{INTRODUÇÃO}

A velocidade autosselecionada (VAUS) é caracterizada como um ritmo de caminhada escolhido livremente pelo sujeito, sendo esta a mais confortável que outras velocidades de caminhada. Em sujeitos sem restrições de caminhada, a VAUS coincide com a velocidade ótima (VOT), entre 4 e 4,5 km. $\mathrm{h}^{-1}$. Nessa velocidade o sujeito apresenta menor custo de transporte $(C)$ que é o consumo de oxigênio $\left(\mathrm{VO}_{2}\right)$ por metro percorrido ${ }^{1}$. Pesquisas a respeito da mecânica e a energética da locomoção, utilizam a esteira rolante para a realização dos seus experimentos ${ }^{2-8}$. Em sujeitos com alterações do padrão da caminhada, como paralisados cerebrais ${ }^{9}$ e sujeitos com acidente vascular encefálico ${ }^{10}$ há diferença entre a velocidade no solo, na esteira rolante e entre a VAUS e a VOF. No entanto, em amputados, a diferença entre a VAUS de caminhada no solo (VAUSS) e na esteira rolante (VAUSE) permanece pouco conhecida.

A determinação da velocidade de caminhada torna-se importante nos estudos de locomoção, pois os parâmetros metabólicos e mecânicos são dependentes desta. Isto ocorre devido às características inerciais que parecem ser diferentes em amputados, bem como às repercussões destas nos parâmetros biomecânicos da caminhada 
destes indivíduos ${ }^{11}$. Um parâmetro analisado e que pode influenciar a dinâmica da caminhada e a escolha da velocidade de caminhada é a estabilidade dinâmica. Existem diferentes formas de obtermos esta variável, entre elas o coeficiente de Lyapunov e a variabilidade dos parâmetros espaço temporais ${ }^{12}$. Em amputados, sabe-se que a mecânica da caminhada possui um padrão assimétrico, como por exemplo, nos tempos de apoio e de balanço. Isso porque, a fase de balanço no membro protético é maior quando comparado ao membro intacto. Já a fase de apoio é menor no membro protético do que no membro contra lateral ${ }^{13}$. Porém, os resultados da estabilidade dinâmica ainda não foram associados com as variáveis metabólicas.

Além das variáveis citadas, a análise possui relação direta com a escolha da velocidade de caminhada. Parece que amputados possuem maior dispêndio energético durante a caminhada e isso pode estar associado com o nível de amputação ${ }^{14-16}$. O C em amputados transfemorais, também atinge valores maiores quando comparado a controles. Logo, a hipótese do estudo foi que a escolha da VAUS em amputados transfemorais será influenciada pela esteira rolante e que esta escolha acarretará em uma menor estabilidade dinâmica. Também foi hipotetizado que através da análise da variável C e do número de Froude, será possível observar diferença entre a VOT e a VAUSE.

\section{MÉTODOS}

\section{Amostra}

Participaram do estudo 10 sujeitos com amputação transfemoral unilateral e 10 sujeitos sem amputações. Os indivíduos amputados utilizavam a prótese de 12-24 meses (média de 17 meses). É importante dizer que os indivíduos foram selecionados a partir de uma clínica de reabilitação de amputados. Foram incluídos no estudo pacientes com amputação transfemoral de origem traumática, devidamente adaptados a prótese, encaixe a vácuo ou soquete prótese de silicone com joelho protético (controle hidráulico) e pé de fibra de carbono, sem lesões ou pressão excessiva sobre o membro residual, adaptado a caminhar na esteira, sem distúrbio neurológico, dificuldades de compreensão, dor ao deambular e sem histórico de doenças cardiopulmonares, neurológicas ou musculoesqueléticas. Este nível de amputação foi escolhido, porque parece apresentar maiores alterações nos padrões mecânicos ${ }^{17}$. Este estudo de corte transversal seguiu as normas do conselho nacional de saúde (446/2012), todos participantes assinaram o termo de consentimento livre e esclarecido, sob a aprovação 2007924 obtida junto ao Comitê de Ética e Pesquisa da Instituição.

\section{Componentes protéticos}

Os componentes protéticos utilizados foram: encaixe com apoio isquiático [CAT-CAM (Contoured Adducted Trochanteric - Controlled Alignment Methord/ USA) encaixe com contenção isquiática], joelho hidráulico (Otto Bock 3R80 - Alemanha) isquiático (CAT-CAM encaixe com contenção isquiática), joelho hidráulico (Otto Bock 3R80) e pé em fibra de carbono. $\mathrm{O}$ alinhamento dos componentes protéticos foi realizado por um protesista, que seguiu os procedimentos convencionais da prática clínica, já descritos em detalhes anteriormente ${ }^{18}$. Previamente à coleta, foi realizado o alinhamento estático da prótese, neste momento foram colocadas todas as angulações adequadas nas articulações protéticas e logo após uma avaliação subjetiva da caminhada. Na Tabela 1 estão descritas as características da amostra e o valor do p para a comparação entre o grupo de controles e o grupo de amputados transfemorais.

\section{Protocolo da velocidade auto selecionada de caminhada}

O protocolo iniciou com a determinação da VAUS no solo. Foi solicitado que o sujeito caminhasse na velocidade mais confortável através de um corredor de $15 \mathrm{~m}$ e o tempo do deslocamento foi cronometrado. Cada sujeito fez o percurso três vezes e foi calculada a média dos valores. O tempo que o indivíduo levou para realizar o percurso foi incluído na equação $d=v t$, (onde d é a distância, $v$ é a velocidades e $t$ é o tempo) que posteriormente calculada resultou na VAUS no solo.

Posteriormente, foi solicitado que o sujeito caminhasse na VAUS na esteira rolante (da marca BH H fitness - Explorer ProAction - sem inclinação - Espanha). A VAUS na esteira rolante foi determinada conforme previamente descrito ${ }^{19}$ e definida como uma velocidade confortável para a caminhada.

\section{Equação da velocidade ótima}

Para a obtenção da VOT, foi utilizada a equação referente ao número de Froude 20 .

$$
F r=v^{2} / g L
$$

onde, Fr é o número de Froude, vé a velocidade $\left(\mathrm{m} \cdot \mathrm{s}^{-1}\right), \mathrm{g}$ é a aceleração da gravidade $\left(9,81 \mathrm{~m} . \mathrm{s}^{-2}\right)$, L é o comprimento do membro inferior (medida do trocânter maior até o solo (em metros).

Na segunda equação foi utilizado o valor de 0,25 para o número de Froude, que equivale a VOT conforme descrito na literatura20,21.

\section{Estabilidade dinâmica}

Foi observada através da variabilidade dos parâmetros espaço temporais da caminhada (tempo e comprimento da passada), obtidos pelos dados de cinemetria. Foi utilizada uma câmera posicionada no plano sagital durante a caminhada na esteira rolante, dois marcadores reflexivos localizados no calcâneo e na base do quinto metatarso. Os dados foram analisados no software Dvideow (Laboratório de Biomecânica - Instituto de Computação - UNICAMP, Campinas, Brasil)22 e calculados em ambiente MatLab 7.1 (Mathworks Inc., USA). A estabilidade dinâmica foi considerada como o coeficiente de variação das variáveis espaço temporais da caminhada obtidas na esteira rolante ${ }^{23}$.

Tabela 1. Características dos sujeitos. Os amputados apresentaram a VAUSE menor que a VAUSS.

\begin{tabular}{|c|c|c|c|}
\hline & $\begin{array}{c}\text { Controles } \\
(n=10)\end{array}$ & $\begin{array}{c}\text { Amputados } \\
(n=10)\end{array}$ & p \\
\hline \multicolumn{4}{|l|}{ Antropométricos } \\
\hline Idade (anos) & $23,1 \pm 2,9$ & $22,3 \pm 4,7$ & 0,08 \\
\hline Massa $(\mathrm{kg})$ & $71,6 \pm 6,5$ & $73,3 \pm 15,0$ & 0,8 \\
\hline Estatura $(\mathrm{cm})$ & $1,78 \pm 0,06$ & $1,78 \pm 0,1$ & 0,76 \\
\hline Comprimento de perna $(\mathrm{cm})$ & $92,8 \pm 4,03$ & $89,9 \pm 5,5$ & 0,056 \\
\hline Comprimento do membro residual $(\mathrm{cm})$ & - & $35 \pm 6$ & - \\
\hline Núemro de Froude & $0,2 \pm 0,02$ & $0,15 \pm 0,03$ & 0,050 \\
\hline \multicolumn{4}{|l|}{ Velocidade de caminhada } \\
\hline VOF Froude $\left(\mathrm{km} \cdot \mathrm{h}^{-1}\right)$ & $5,4 \pm 0,04$ & $5,2 \pm 0,05$ & 0,060 \\
\hline VAUSE esteira $\left(\mathrm{km} \cdot \mathrm{h}^{-1}\right)$ & $4,0 \pm 0,3$ & $3,0 \pm 0,41$ & 0,043 \\
\hline VAUSS solo $\left(\mathrm{km} \cdot \mathrm{h}^{-1}\right)$ & $4,0 \pm 0,9$ & $3,8 \pm 0,39$ & 0,050 \\
\hline \multicolumn{4}{|l|}{ Estabilidade Dinâmica } \\
\hline COV comprimto da passada & $5,3 \pm 0,28$ & $12,5 \pm 1,61$ & 0,005 \\
\hline COV tempo da passada & $5,5 \pm 0,29$ & $8,2 \pm 0,62$ & 0,04 \\
\hline \multicolumn{4}{|l|}{ Variáveis Metabólicas } \\
\hline $\mathrm{VO}_{2}\left(\mathrm{ml}^{\prime} \mathrm{kg}^{-1} \cdot \mathrm{min}^{-1}\right)$ & $1,93 \pm 0,53$ & $3,95 \pm 0,47$ & 0,004 \\
\hline Custo de transporte $\left(\mathrm{J} \cdot \mathrm{kg}^{-1} \cdot \mathrm{m}^{-1}\right)$ & $1,69 \pm 0,43$ & $4,78 \pm 0,94$ & 0,001 \\
\hline
\end{tabular}

Dados em média \pm DP. VOF Froude: velocidade ótima de caminhada calculada através do número de Froude, VAUSE: velocidade auto selecionada de caminhada mensurada na esteira rolante; VAUSS: velocidade auto selecionada mensurada no solo, Cov comprimento da passada: coeficiente de variaçäo do comprimento da selecionada mensurada no solo, Cov comprimento da passada: coeficiente de variaçáo do comprimento da
passada da esteira rolante, Cov tempo da passada: coeficiente de variação do tempo da passada da esteira rolante, $\mathrm{VO}_{2}$ : consumo de oxigênio da velocidade auto selecionada da esteira rolante, Custo de transporte da velocidade auto selecionada na esteira rolante. 


\section{Protocolo do custo de transporte}

O protocolo teve início com a mensuração do consumo de oxigênio em repouso na posição ortostática durante cinco minutos. Após, o individuo caminhou em sua VAUS durante cinco minutos na esteira rolante. Foi realizada análise de gases durante todo o protocolo na esteira (analisador de gases VO2000) ${ }^{19}$.

\section{Análise custo de transporte}

A média dos valores de relativo a partir do terceiro minuto da caminhada na esteira foi subtraído pelo valor do pré exercício (em ortostase) e, então, os valores foram convertidos para Joules (J). Para isso o valor foi dividido por 60 segundos. Posteriormente, os dados foram divididos pela velocidade em $\mathrm{m} \cdot \mathrm{s}^{-1}$ para determinar o $\mathrm{C}$ da caminhada em J.kg-1 $\mathrm{m}^{-1)} 19$.

Para comparar a VAUSS, VAUSE eVOF foi utilizada uma ANOVA de dois fatores seguido de um post hoc de Tukey. Os dados foram considerados significativos com $p \leq 0.05$.

\section{RESULTADOS}

Conforme apresentado na Tabela 1 o número de Froude foi maior para o grupo de controles $(P=0.05)$. A estabilidade dinâmica apresentou maior variabilidade para o comprimento $(P=0.005)$ e o tempo $(\mathrm{P}=0.040)$ da passada para os amputados. $\mathrm{O}$ e o $\mathrm{C}$ foram maiores para os amputados ( $\mathrm{P}=0.004 ; \mathrm{P}=0.001$ respectivamente).

A Figura 1 mostra os valores da média e desvio padrão da amostra para as três medidas de velocidade para cada grupo. A VOF foi maior que a VAUSS eVAUSE ( $P=0.001 ; P=0.001$ respectivamente) para os controles e amputados ( $P=0.004 ; P=0.001$ respectivamente). Para o grupo controle não houve diferença entre a VAUSE e a VAUSS. Os amputados apresentaram a VAUSE menor que a VAUSS ( $P=0.021)$.

\section{DISCUSSÃO}

A diferença encontrada entre a VAUSE e a VAUSS para amputados transfemorais (Tabela 1, Figura 1 representa as diferenças), pode auxiliar tanto em pesquisas como na reabilitação clínica destes sujeitos. Apesar da diferença entre as duas medidas, as análises biomecânicas e fisiológicas são conduzidas na esteira por serem realizadas em ambiente de laboratório. No entanto, sugerimos que em pesquisas futuras, essa diferença seja considerada na elaboração do protocolo.

Além das diferenças encontradas quanto a VAUS, também foi observada diferença na análise biomecânica da estabilidade dinâmica entre os grupos. Sabe-se que o mecanismo compensatório da

Velocidade autosselecionada na esteira e no solo, e velocidade ótima estimada pelo número de Froud para o grupo de controles e o grupo de amputados.
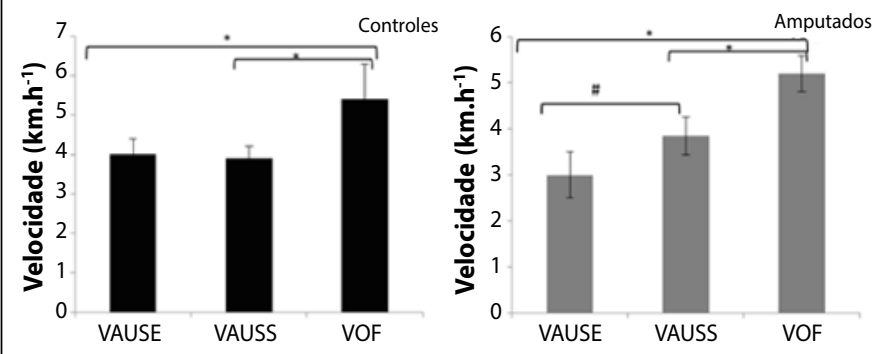

Figura 1. média \pm DP das diferentes velocidades, denominadas velocidade auto selecionada na esteira (VAUSE), velocidade auto selecionada no solo (VAUSS), velocidade ótima estimada pelo número de Froude (VOF). * e \# representam diferença significativa, * para diferença intra grupo, para controles (esquerda) e para amputados (direita), entre a VOF e a VAUSE, e entre a VOF e a VAUSS. \# representa diferença intra grupo para os amputados entre a VAUSE e a VAUSS. marcha de amputados transfemorais é uma importante adaptação para sua estabilidade ${ }^{24}$ sendo que as possíveis assimetrias das forças verticais e horizontais da perna protética são compensados pela perna intacta na caminhada de amputados em diferentes velocidades de caminhada ${ }^{25} \mathrm{~A}$ diferença nos padrões da estabilidade também pode ter ocorrido devido a maior variabilidade dos parâmetros espaço temporais no grupo dos indivíduos amputados, quando comparados com o grupo controle. Todavia questões como o correto ajuste da prótese e alinhamento protético são essenciais para estabilidade e estratégias adaptativas da marcha de amputados ${ }^{26}$.

Os resultados das variáveis metabólicas (e C) corroboram com as variáveis mecânicas. Porém, ao contrário do que acontece com indivíduos sem restrições, os indivíduos amputados ${ }^{1,27}$ apresentaram valores maiores para VAUS e C. Ao observar a Figura 2 podemos sugerir que o $\mathrm{C}$ diminui à medida que a velocidade aumenta ${ }^{4}$.

Os valores de $\mathrm{VO}_{2}$ foram superiores quando comparados aos controles, isso pode ter ocorrido em função da menor estabilidade dinâmica. Como a VAUS dos amputados transfemorais foi menor, pode ter influenciado o $C$, e esta escolha talvez tenha contribuído para uma maior instabilidade dinâmica deste grupo. Estudo recente destaca que o tamanho do membro residual parece não afetar a energia metabólica e também não afeta o deslocamento do centro de massa corporal de amputados transfemorais ${ }^{28}$.

As limitações do estudo foram quanto a dificuldade da aplicação metodológica da análise da cinemetria e do analisador de gases fora da esteira rolante, desta forma não foi possível comparar as variáveis mecânica (estabilidade dinâmica) e metabólicas (C e ) nas duas situações. Mesmo assim, consideramos que os resultados deste estudo são relevantes, pois podem auxiliar tanto na prática clínica como em elaborações de protocolos de pesquisas na área.

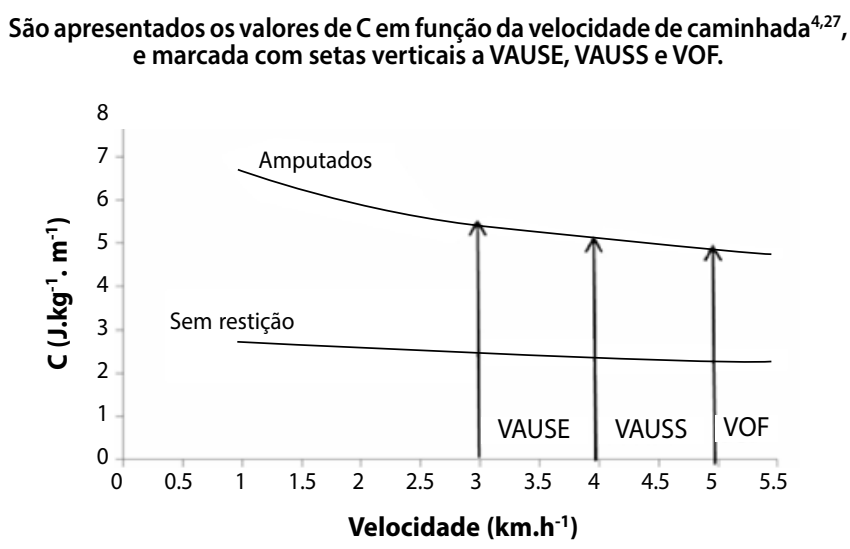

Figura 2. C: custo de transporte (J.kg-1.m-1), em função da velocidade (km.h-1). Na linha cinza os valores de $C$ de amputados transfemorais4 e na linha inferior os valores para indivíduos sem restrições 27. A seta VAUSE é referente à velocidade preferida na esteira, a seta VAUSS é a velocidade preferida no solo, a seta VOF é a velocidade ótima pelo número de Froude.

\section{CONCLUSÃO}

A VAUSE e a VAUSS em amputados transfemorais foram diferentes, conforme as hipóteses do presente estudo. A VOF também é diferente da VAUS em amputados transfemorais o que concorda com o maior $C$ encontrado nesta população. Uma variável que pode auxiliar o entendimento dessa disparidade é a variação nos parâmetros cinemáticos, como a estabilidade dinâmica, apresentada neste estudo.

Todos os autores declararam não haver qualquer potencial conflito de interesses referente a este artigo. 
CONTRIBUIÇÕES DOS AUTORES: Cada autor contribuiu individual e significativamente para o desenvolvimento do manuscrito. RLB (0000-0003-4343-7336)* iniciou a abordagem ao problema de pesquisa e participou de todas etapas: construção do projeto, coleta de dados, análise dos dados, discussão dos resultados e escrita do manuscrito. NAG (0000-0002-7282-7272)* e ABS (0000-0003-0515-0166)* participaram de todas as etapas, desde a construção do projeto, coleta de dados, análise dos dados, discussão dos resultados e escrita do manuscrito. JS (0000-0002-6182-8560** participou das coletas de dados, análise dos dados, discussão dos resultados e escrita do manuscrito. PFS (0000-0002-5756-2675)* realizou a pesquisa bibliográfica e a revisão do manuscrito. LAT (0000-0003-1742-5016)* participou principalmente da discussão dos resultados e escrita do manuscrito. Todos os autores contribuíram de forma intensa no estudo. *ORCID (Open Researcher and Contributor ID).

\section{REFERÊNCIAS}

1. Saibene F, Minetti A E. Biomechanical and physiological aspects of legged locomotion in humans. Eur J Appl Physiol. 2003;88(4-5):297-316.

2. Genin JJ, Bastien GJ, Franck B, Detrembleur C, Willems P. Effect of speed on the energy cost of walking in unilateral traumatic lower limb amputees. Eur J Appl Physiol. 2008;103(6):655-63.

3. Traballesi M, Porcacchia P, AvernaT, Brunelli S. Energy cost of walking measurements in subjects with lower limb amputations: a comparison study between floor and treadmill test. Gait Posture. 2008;27(1):70-5.

4. Detrembleur C, Vanmarsenille JM, Cuyper F, Dierick F. Relationship between energy cost, gait speed, vertical displacement of centre of body mass and efficiency of pendulum-like mechanism in unilateral amputee gait. Gait Posture. 2005;21(3):333-40.

5. Selles RW, Korteland S, Van Soest AJ, Bussmann JB, Stam HJ Lower-leg inertial properties in transtibial amputees and control subjects and their influence on the swing phase during gait. Arch Phys Med Rehabil. 2003;84(4):569-77.

6. Schmalz T, Blumentritt S, Jarasch R. Energy expenditure and biomechanical characteristics of lower limb amputee gait: the influence of prosthetic alignment and different prosthetic components. Gait Posture. 2002;16(3):255-63.

7. Isakov E, Burger H, Krajnik J, Gregoric M, Marincek C. Influence of speed on gait parameters and on symmetry in trans-tibial amputees. Prosthet Orthot Int. 1996;20(3):153-8.

8. Murtagh EM, Boreham CA, Murphy MH.. Speed and exercise intensity of recreational walkers. Prev Med. 2002;35(4):397-400

9. van den Hecke A1, Malghem C, Renders A, Detrembleur C, Palumbo S, Lejeune TM. Mechanical work, energetic cost, and gait efficiency in children with cerebral palsy. J Pediatr Orthop. 2007;27(6):643-7

10. Olney SJ, Richards C. Hemiparetic gait following stroke. Part I: Characteristics. Gait Posture. 1996;11(4):136-48,

11. Santos AB, Bona RL, Castro FAS, Tartaruga, LAP. Aspectos biomecânicos e fisiológicos da fadiga na locomoção humana: conceitos, mecanismos e aplicações. Cien Movimento. 2010;23(1):89-98.

12. Hausdorff JM. Gait variability: methods, modeling and meaning. J Neuroeng Rehabil. 2005;2:19.

13. Winter DA, Sienko SE. Biomechanics of below-knee amputee gait. J Biomech.1988;21(5):361-7.

14. Waters RL, Perry J. Energy cost of walking of amputees: the influence of level of amputation. J Bone Joint Surg Am. 1976;58(1):42-6.
15. Bona RL, Ribeiro J, Aldabe D. Avaliação do gasto energético em pacientes amputados de membro inferior protetizados. Arq Sanny Pesq Saúde. 2008;1(2):98-108

16. Bona RL, Tartaruga LAP. Mecânica e energética da locomoção de amputados: uma revisão. Pensar a Prática. 2011;14(1):1-14.

17. Cheung C, Wall JC, Zelin S. A microcomputer-based system for measuring temporal asymmetry in amputee gait. Prosthet Orthot Int. 1983;7(3):131-40.

18. Sin SW, Chow DH, Cheng JC. Significance of non-level walking on transtibial prosthesis fitting with particular reference to the effects of anterior-posterior alignment. J Rehabil Res Dev. 2001;38(1):1-6.

19. Figueiredo P, Ribeiro PA, Bona RL, Peyré-Tartaruga LA, Ribeiro JP. Ventilatory determinants of self-selected walking speed in chronic heart failure. Med Sci Sports Exerc. 2013;45(3):415-9.

20. Alexander RM. Optimization and gaits in the locomotion of vertebrates. Physiol Rev. 1989;69(4):1199-227.

21. Vaughan $\mathrm{CL}, \mathrm{O}$ 'Malley MJ. Froude and the contribution of naval architecture to our understanding of bipedal locomotion. Gait Posture. 2005;21(3):350-62.

22. Barros RM, Brenzikofer R, Leite NJ, Figueroa PJ. Desenvolvimento e avaliação de um sistema para análise cinemática tridimensional de movimentos humanos. Rev. Bras. Eng. Bioméd. 1999;15(1-2):79-86.

23. Hausdorff JM, Rios DA, Edelberg HK. Gait variability and fall risk in community-living older adults: a 1-year prospective study. Arch Phys Med Rehabil. 2001;82(8):1050-6

24. Drevelle $X$, Villa $C$, Bonnet $X$, Loiret I, Fodé $P$, Pillet H. Vaulting quantification during level walking of transfemoral amputees. Clin Biomech. 2014;29(6):679-83.

25. Schaarschmidt M, Lipfert SW, Meier-Gratz C, Scholle HC, Seyfarth A. Functional gait asymmetry of unilateral transfemoral amputees. Hum Mov Sci. 2012;31(4):907-17.

26. Kendell C, Lemaire ED, Kofman J, Dudek N-Gait adaptations of transfemoral prosthesis users across multiple walking tasks. Prosthet Orthot Int. 2016;40(1):89-95.

27. Mian OS, Thom JM, Ardigo LP, Narici MV, Minetti AE. Metabolic cost, mechanical work, and efficiency during walking in young and older men. Acta Physiol. 2006;186(2):127-39.

28. Bell JC, Wolf EJ, Schnall BL, Tis JE, Potter BK. Transfemoral amputations: is there an effect of residual limb length and orientation on energy expenditure? Clin Orthop Relat Res. 2014;472(10):3055:61 